\title{
Outcomes of traumatic brain injury: the prognostic accuracy of various scores and models
}

\author{
Jose D. Charry ${ }^{1}$, Sandra Navarro-Parra ${ }^{1}$, Juan Solano ${ }^{1}$, Luis Moscote-Salazar ${ }^{1}$, \\ Miguel Angel Pinzón ${ }^{2}$, Jorman Harvey Tejada ${ }^{2}$ \\ ${ }^{1}$ Fundación Universitaria Navarra - UNINAVARRA, Neiva, Colombia \\ ${ }^{2}$ School of Medicine, Universidad Surcolombiana, Neiva, Colombia
}

\begin{abstract}
Introduction. Traumatic Brain Injury (TBI) is a worldwide health problem, and is a pathology that causes significant mortality and disability in Latin America. Different scores and prognostic models have been developed in order to predict the neurologi-cal outcomes of patients. We aimed to test the prognostic accuracy of the Marshall CT classification system, the Rotterdam CT scoring system, and the IMPACT and CRASH models, in predicting 6-month mortality and 6-month unfavourable outcomes in a cohort of trauma patients with TBI in a university hospital in Colombia.

Methods. We analysed 309 patients with significant TBI who were treated in a regional trauma centre in Colombia over a two year period. Bivariate and multivariate analyses were undertaken. The discriminatory power of each model, as well as its accu-racy and precision, were assessed by logistic regression and AUC. Shapiro Wilks, chi2 and Wilcoxon test were used to compare the actual outcomes in the cohort against the predicted outcomes.

Results. The median age was 32 years, and $77.67 \%$ were male. All four prognostic models showed good accuracy in predicting outcomes. The IMPACT model had the greatest accuracy in predicting an unfavourable outcome (AUC 0.864; $95 \% \mathrm{Cl} 0.819-0.909$ ) and in predicting mortality (AUC $0.902 ; 95 \% \mathrm{Cl} 0.862-0.943$ ) in patients with TBI.

Conclusion. All four prognostic models are applicable to eligible TBI patients in Colombia. The IMPACT model was shown to be more accurate than the other prognostic models, and had a higher sensitivity in predicting 6-month mortality and 6month unfavourable outcomes in patients with TBI in a university hospital in Colombia.
\end{abstract}

Key words: traumatic brain injury, prognosis models, neurological outcome (Neurol Neurochir Pol 2019; 53 (1):55-60)

\section{Introduction}

Traumatic brain injury (TBI) has a significant impact worldwide. Its incidence has been reported to be nearly 200 cases per 100,000 people worldwide [1]. According to the Global Burden of Disease Study published in 2010 by the World Health Organisation, trauma remains a public health problem and represents an important burden of disease for healthcare systems in Latin American countries [2-3]. In Colombia, the burden of such injuries particularly affects the male, economically active population aged between 12 and 45 years. In 2013, 26,000 deaths were due to trauma, and most of these were associated with interpersonal violence. Of these injuries, a large percentage was associated with closed TBI and penetrating TBI [4].

TBI remains the main cause of death and disability in young adults worldwide [5-6]. It is a heterogeneous disease in relation to cause, pathology, severity, and prognosis. This results in considerable uncertainty regarding the expected outcome of individual patients. Several outcome prediction models have been developed for the prognosis of TBI patients to address this uncertainty [7]. These prognostic models can be used to combine different characteristics of individual patients to predict their clinical outcome. Prognostic models may also be useful as tools to compare outcomes across institutions, healthcare systems, and countries, and may be

Address for correspondence: Jose D. Charry, Fundación Universitaria Navarra - Uninavarra, Calle 10 No. 6-41, Neiva - Colombia, e-mail: danielcharry06@gmail.com, phone: (+57) 3158019678 
an essential part of the planning of new studies in the field of brain injury.

The Glasgow Outcome Scale (GOS) is widely used in TBI management and surgery outcome studies [8]. Two prognostic models (the International Mission for Prognosis and Analysis of Clinical Trials in TBI [IMPACT] model, and the Corticosteroid Randomisation After Significant Head Injury [CRASH] model), both based on large TBI clinical trial datasets, have recently been cross-validated and externally validated. [9-12].

IMPACT applies to adult patients (age $\geq 14$ years) with a Glasgow Coma Scale (GCS) score of $\leq 12$ to predict the probabilities of 6-month favourable outcome and 6-month mortality.

CRASH applies to adult patients (age $\geq 16$ years) with a GCS score of $\leq 14$ to predict the probabilities of 14 -day mortality and 6-month unfavourable outcome.

The calculator models predict outcomes based on specific variables including country of incident, patient age, and clinical and imaging findings in the emergency room [13-14].

Another diagnostic technique for assessing TBI is brain imaging by computed tomography (CT) and magnetic resonance imaging (MRI). Brain imaging significantly aids early diagnosis and effective treatment of life-threatening conditions in patients with TBI [15]. However, brain CT is the gold standard for assessing patients with acute TBI. Currently, there are two CT-based systems for evaluating CT findings, the Marshall Classification System (MCS) and the Rotterdam Scoring System (RSS) [16-17].

The MCS, developed by Marshall et al. in 1991, was the first CT-based system for determining the prognosis of TBI. The MCS classifies CT findings into four grades: Grade 1, no pathologic findings; Grade 2, basal cisterns are present and midline shift is less than $5 \mathrm{~mm}$; Grade 3, basal cisterns are compressed; and Grade 4, midline shift is greater than $5 \mathrm{~mm}$. [16]. This system was developed primarily for predicting patient outcomes and the risk for increased intracranial pressure in patients with severe TBI.

In 2005 Maas et al. introduced the RSS. This system provides a better estimation of disease prognosis by using certain criteria such as basal cistern condition, midline shift, traumatic subarachnoid or intraventricular haemorrhage, and epidural haematoma. Rotterdam scores predict post trauma 6-month mortality rate as follows: score 1, 5\%; score 2, 7\%; score $3,16 \%$; score $4,26 \%$; score $5,53 \%$; and score $6,61 \%$.

In this study, we aimed to test the prognostic accuracy of the Marshall CT score and the Rotterdam CT score, as well as the accuracy of the International Mission for Prognosis and Analysis of Clinical Trials in Traumatic Brain Injury (IMPACT) and Corticosteroid Randomisation after Significant Head Injury (CRASH) models, in predicting 14-day mortality, 6-month mortality, and 6-month unfavourable outcomes in a cohort of trauma patients with TBI in a university hospital in Colombia.

\section{Materials and methods}

\section{Patient population}

This retrospective, observational cohort study was performed at Neiva University Hospital (NUH) in southern Colombia. Included in the study were patients with TBI who were admitted to NUH between January 2014 and December 2015. Approval was obtained from the NUH quality improvement office and the Ethical Committee of Neiva University Hospital prior to conducting this study.

NUH is a 504-bed, level I trauma centre and tertiary referral hospital in southern Colombia that admits approximately 2,000 adult trauma patients per year and has 30 adult ICU beds. This hospital is the primary trauma centre for 3.2 million inhabitants living in a region extending over 60,000 square miles. In order to be included in this study, patients had to be 18 years or older and suffering from TBI (loss of consciousness or change in consciousness level with a head and neck Abbreviated Injury Severity score $\geq 2$ ). The Glasgow Coma Scale (GCS) was used to classify the trauma level: minor (GCS 15-13), moderate (GCS 9-12), or severe (GCS 3-8).

\section{Clinical outcome}

The variables and results were evaluated according to the Glasgow Outcome Scale (GOS) at 6 months post injury, creating a dichotomous variable with a favourable outcome (GOS 4 or 5), and an unfavourable outcome (GOS 1-3) $[11,12]$. In addition, we evaluated the prediction of mortality that was given by the Marshall CT score, Rotterdam CT score, and the IMPACT and CRASH models versus those obtained in the study patients.

\section{Prediction of outcome}

We used the web-based prognosis calculator published by the CRASH collaborators and applied it to the patient group described above. The variables which were found by the CRASH study group to have the largest influence on the outcome, and were thus used in the CRASH prognostic calculator, were: country, age, Glasgow Coma Scale (GCS) score, pupil reactivity, and the presence of any other major trauma (defined as an injury which would have rendered the patient in need of in-hospital care in and of itself). If a CT scan of the brain was performed within eight hours of trauma, the following features on the scan were used in the calculation of prognosis: presence of petechial haematoma, obliteration of basal cisterns or the third ventricle, subarachnoid haemorrhage, midline shift, and non-evacuated haematoma. For the individual patient, the CRASH prognosis calculator predicts risk of mortality at 14 days after the injury and the risk of an unfavourable outcome (GOS 1-3) at 6 months after trauma with percentages at $95 \%[10]$.

The Marshall Classification System (MCS) was the first CT classification system to determine the prognosis of TBI. 
The MCS classifies CT findings into four grades: Grade 1, no pathologic findings; Grade 2, basal cisterns are present and midline shift is less than $5 \mathrm{~mm}$ or presence of high density lesions or mixed of no more than $25 \mathrm{cc}$; Grade 3, basal cisterns are compressed with deviation of midline of no more than $5 \mathrm{~mm}$; and Grade 4, midline shift is greater than $5 \mathrm{~mm}$; mass evacuated and not evacuated mass. This system was developed primarily for predicting patient outcomes and the risk for increased intracranial pressure in patients with severe TBI [15].

The IMPACT model is an analysis that has been used for the development of prognosis models for mortality and unfavourable outcomes at six months in patients with moderate or severe TBI. In our study, we only included patients with severe TBI (Glasgow Coma Scale $\leq 8$ ) on admission. Using IMPACT, the following criteria were evaluated: age, motor score, pupil reactivity, presentation of states of hypoxia, hypotension, Marshall CT classification, subarachnoid bleeding on $\mathrm{CT}$, epidural mass on $\mathrm{CT}$ and laboratory tests such as glucose levels and haemoglobin [9-11].

The Rotterdam Scoring System (RSS) provides a better estimation of disease prognosis by using certain criteria such as basal cistern condition, midline shift, traumatic subarachnoid or intraventricular haemorrhage, and epidural haematoma. Rotterdam scores predict post trauma 6-month mortality rate as follows: score 1, 5\%; score 2, 7\%; score 3, 16\%; score 4, $26 \%$; score 5, 53\%; and score 6, 61\% [16].

\section{Statistics}

Values are reported as mean \pm standard deviation. Discrete variables are reported as median and range. Logistic fit and receiver operating characteristic (ROC) statistics were used as indicated. The statistical software used was SPSS Statistics (Version 21, IBM Corporation) and the R software environment (Version 2.15.2, The R Foundation for Statistical Computing, Vienna, Austria). A p value $<0.05$ was regarded as significant. The discriminatory power of the model, its accuracy and precision, were assessed by logistic regression and as the area under the receiver operating characteristic curve (AUC). Shapiro Wilks, chi2 and the Wilcoxon test were used to compare real outcomes in the cohort against predicted outcomes. We made a multivariate logistic regression and analysis. We excluded variables that were not significant at a 5\% level. We quantified each variable's predictive contribution by its z score (the model coefficient divided by its standard error). We explored linearity and interactions between the variables and all predictors were evaluated by $\mathrm{p}$ value and the IC.

\section{Results}

309 patients were admitted with a diagnosis of TBI over a period of two years to Neiva University Hospital. Median age in the validation cohort was 32 years and $77.67 \%$ were male. $95.47 \%$ of the patients had experienced blunt trauma, the median of injury severity score was 4 , imaging findings showed $23.3 \%$ had midline shift, and subdural haematoma was present in $28.8 \%$. The characteristics of the 309 patients included in this study are set out in Table 1.

Six-month mortality was $12.62 \%$. Six-month unfavourable outcome was $17.15 \%$. The IMPACT model had the best rate of accuracy in predicting 6-month unfavourable outcome was $20 \% \mathrm{p}<0.001$ (AUC $0.864 ; 95 \%$ CI $0.819-0.909$ ) and 6-month mortality was $12 \% \mathrm{p}<0.001$ (AUC $0.902 ; 95 \% \mathrm{CI}$ $0.862-0.943)$ in patients with TBI. Mortality prediction by Marshall CT score was $13.5 \% \mathrm{p}<0.001$ (AUC 0.819; 95\% IC $0.769-0.869)$. The 6 -month mortality prediction by CRASH prognosis calculator was $5.3 \% \mathrm{p}<0.001$ (AUC $0.877 ; 95 \%$ IC $0.825-0.930$ ). The six-month unfavourable outcome prediction by CRASH was $20 \% \mathrm{p}<0.001$ (AUC $0.832 ; 95 \%$ IC $0.785-0.881$ ). The 6 -month mortality prediction by Rotterdam

Table 1. Characteristics of 309 patients with TBI

\begin{tabular}{lc}
\multicolumn{1}{c}{ Variable } & Value \\
\hline Median age in years (IQR) & $32(23-47)$ \\
Number of males (\%) & $240(77.67)$ \\
Median initial GCS score (IQR) & $9(6-15)$ \\
Median ISS score (IQR) & $4(4-16)$ \\
Motor vehicle accidents (\%) & $276(89.32)$ \\
Pupils (\%) & \\
Both nonreactive & $49(15.85)$ \\
One reactive & $10(3.23)$ \\
Both reactive & $250(80.91)$ \\
CT brain appearances (\%) & \\
Effaced basal cistern & $226(73.14)$ \\
Midline shift & $72(23.30)$ \\
Subarachnoid blood & $62(20.06)$ \\
Epidural haematoma & $38(12.30)$ \\
Subdural haematoma & $89(28.80)$ \\
Surgical management, no. (\%) & $126(40.78)$ \\
Drainage of epidural haematoma & $23(18.25)$ \\
Drainage of subdural haematoma & $68(53.96)$ \\
Decompressive craniectomy & $9(7.14)$ \\
ICP monitoring & $26(20.63)$ \\
Unfavourable outcomes (\%) & $53(17.15)$ \\
Favourable outcomes (\%) & $256(82.84)$ \\
Median length of ICU stay, days (IQR) & $12(6-14)$ \\
Median length of acute hospital ward stay, & $26(14-49)$ \\
days (IQR) & \\
Mortalities (\%) & \\
ICU-intensive care unit; IQR - interquartile range; GCS - Glasgow Coma Scale; \\
\end{tabular}


Table 2. Correlation between mortality and predictive models

\begin{tabular}{|c|c|c|c|c|}
\hline Model & Six-month predicted mortality & Six-month mortality & Spearman's rho & $\mathbf{p}$ \\
\hline $\begin{array}{l}\text { Rotterdam CT } \\
\text { score }\end{array}$ & $7 \%$ & & 0.031 & 0.000 \\
\hline $\begin{array}{l}\text { Marshall CT } \\
\text { score }\end{array}$ & $13.5 \%$ & & 0.025 & 0.000 \\
\hline IMPACT & $12 \%$ & $12.62 \%$ & 0.020 & 0.000 \\
\hline CRASH & $5.3 \%$ & & 0.026 & 0.000 \\
\hline Model & $\begin{array}{l}\text { Six-month predicted } \\
\text { unfavourable outcome }\end{array}$ & $\begin{array}{l}\text { Six-month unfavourable } \\
\text { outcome }\end{array}$ & Spearman's rho & $\mathrm{p}$ \\
\hline IMPACT & $20 \%$ & $17.15 \%$ & 0.023 & 0.001 \\
\hline CRASH & $20 \%$ & & 0.024 & 0.001 \\
\hline
\end{tabular}

CT score was 7\% p < 0.001 (AUC 0.875; 95\% IC 0.814-0.936). These results are set out in Table 2 .

Comparing the prediction of mortality and a six-month unfavourable outcome with the observed long-term are in Figure 1.

\section{Discussion}

TBI is a medical and surgical disease of major importance globally. [8] The World Health Organisation predicts that traffic accidents will be the third-leading cause of illness and injuries worldwide by 2020, and this is one of the most common causes of TBI. Prognostication is important when considering outcomes, especially when it's considered to be potentially life-saving. Traditionally, neurosurgeons have relied on individual clinical parameters such as age, initial GCS score, and pupillary responses, combined with a radiological assessment, to guide clinical decisions and when counselling family members and surrogate decision makers regarding prognosis [18].
Different models have been described for predicting mortality and adverse neurological outcomes in TBI patients, the best known of which are the Marshall CT score, the Rotterdam CT score, and the IMPACT and CRASH models. In our cohort study, we had patients who were victims of head trauma and in applying the four models we evaluated the observed versus the predicted outcomes 6 months after the trauma. We found good performance from all four models in predicting mortality. However, the IMPACT model had the best performance in our study. Different studies have been conducted into the validation of these models. In these studies, patients with severe and moderate traumatic brain injury have been evaluated, and use of the different models and scores has shown that their performance and sensitivity is high [19-23].

Studies related to TBI in populations of adolescents, adults and the elderly have revealed the presence of physical and cognitive consequences and inadequate emotional, behavioural, and inappropriate regulation [24]; some have even suggested that they may present a global functional disability [25] and long-term psychosocial disability [26]. Among the
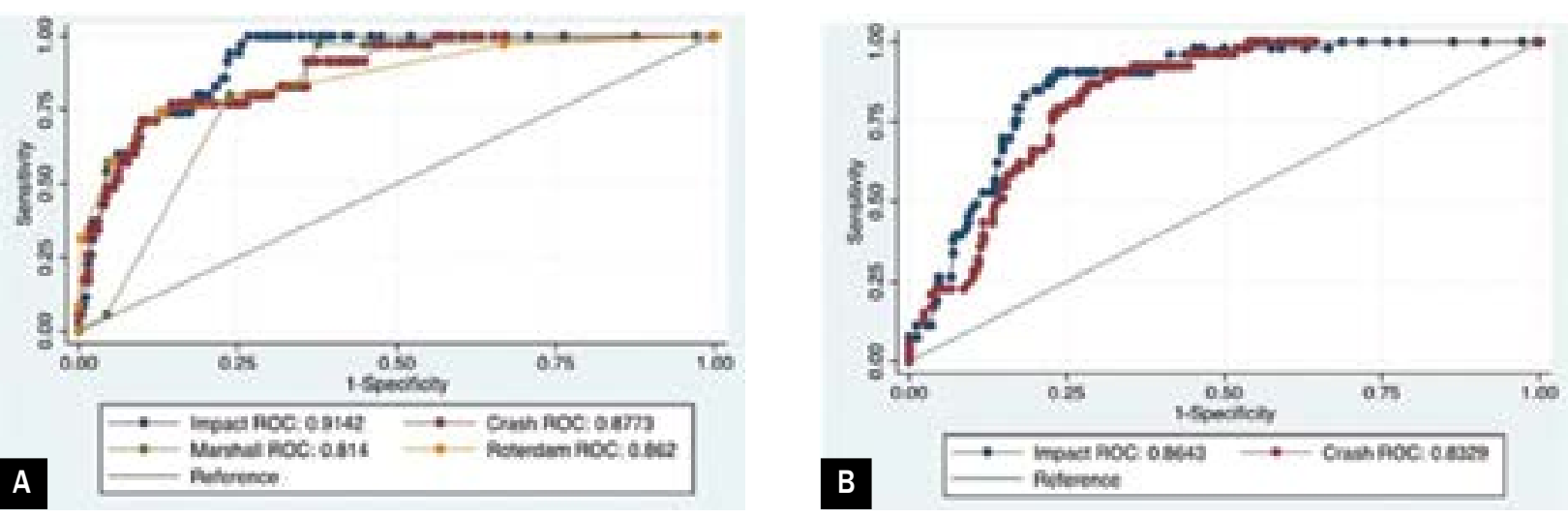

Figure 1. A. AUC of the models for six-month mortality, B. AUC of the models for six-month unfavourable outcome 
survivors of trauma, a considerable proportion are left with important consequences that prevent their return to previous activities, or render impossible academic, professional or social progress [27]. This is why it is essential to implement neurocognitive rehabilitation programmes that favour the processes of adaptation and improvement of the quality of life of both patients and their families. The intervention that has been shown to be the most effective in addressing this type of health event is the non-pharmacological one, framed within a holistic model and a neurobehavioural paradigm that takes into account the results of the neuropsychological assessment and the individual's environment, in addition to adjusting to the needs of the patient and family members. Rehabilitation processes include the more complex processes involved in returning to work [28] and vocational aspects have been shown to be more effective when adapted to the conditions of each individual patient [29].

\section{Conclusions}

The Marshall CT score, Rotterdam CT score, IMPACT and CRASH models were useful in predicting mortality and unfavourable outcomes in TBI patients in a university hospital in Colombia. The IMPACT model was shown to be more accurate than the other prognostic models, and had a higher sensitivity in predicting 6-month mortality and 6-month unfavourable outcomes in patients with TBI.

Conflicts of interest/disclosures. The authors declare that they have no financial or other conflicts of interest in relation to this research and its publication.

\section{References}

1. Byass $P$, de Courten M, Graham WJ, et al. Reflections on the global burden of disease 2010 estimates. PLoS Med. 2013; 10(7): e1001477, doi: 10.1371/journal.pmed.1001477, indexed in Pubmed: 23843748.

2. Horton R. GBD 2010: understanding disease, injury, and risk. Lancet. 2012; 380(9859): 2053-2054, doi: 10.1016/S01406736(12)62133-3, indexed in Pubmed: 23245595.

3. Norton R, Kobusingye 0. Injuries. New England Journal of Medicine. 2013; 368(18): 1723-1730, doi: 10.1056/nejmra1109343.

4. De la. Comportamiento del homicidio en Colombia, 2013. Forensis. ; 2013: 79-125.

5. Maas AIR, Stocchetti N, Bullock R. Moderate and severe traumatic brain injury in adults. Lancet Neurol. 2008; 7(8): 728-741, doi: 10.1016/S1474-4422(08)70164-9, indexed in Pubmed: 18635021.

6. Ghajar J. Traumatic brain injury. The Lancet. 2000; 356(9233): 923929, doi: 10.1016/s0140-6736(00)02689-1.

7. Perel P, Edwards P, Wentz R, et al. Systematic review of prognostic models in traumatic brain injury. BMC Med Inform Decis Mak. 2006; 6: 38, doi: 10.1186/1472-6947-6-38, indexed in Pubmed: 17105661.

8. Jennett B, Bond M. Assessment of outcome after severe brain damage. Lancet. 1975; 1(7905): 480-484, indexed in Pubmed: 46957.
9. Murray G, Butcher I, McHugh G, et al. Multivariable Prognostic Analysis in Traumatic Brain Injury: Results from The IMPACT Study. Journal of Neurotrauma. 2007; 24(2): 329-337, doi: 10.1089/neu.2006.0035.

10. Perel P, Arango M, Clayton T, et al. MRC CRASH Trial Collaborators. Predicting outcome after traumatic brain injury: practical prognostic models based on large cohort of international patients. BMJ. 2008; 336(7641): 425-429, doi: 10.1136/bmj.39461.643438.25, indexed in Pubmed: 18270239.

11. Steyerberg EW, Mushkudiani N, Perel P, et al. Predicting outcome after traumatic brain injury: development and international validation of prognostic scores based on admission characteristics. PLOS Med. 2008; 5(8): e165; discussion e165, doi: 10.1371/journal. pmed.0050165, indexed in Pubmed: 18684008.

12. Roozenbeek B, Lingsma HF, Lecky FE, et al. International Mission on Prognosis Analysis of Clinical Trials in Traumatic Brain Injury (IMPACT) Study Group, Corticosteroid Randomisation After Significant Head Injury (CRASH) Trial Collaborators, Trauma Audit and Research Network (TARN). Prediction of outcome after moderate and severe traumatic brain injury: external validation of the International Mission on Prognosis and Analysis of Clinical Trials (IMPACT) and Corticoid Randomisation After Significant Head injury (CRASH) prognostic models. Crit Care Med. 2012; 40(5): 1609-1617, doi: 10.1097/ CCM.0b013e31824519ce, indexed in Pubmed: 22511138.

13. Honeybul S, Ho KM, Lind CRP, et al. The retrospective application of a prediction model to patients who have had a decompressive craniectomy for trauma. J Neurotrauma. 2009; 26(12): 2179-2183, doi: 10.1089/neu.2009.0989, indexed in Pubmed: 19645531.

14. Honeybul S, Ho KM, Lind CRP, et al. Observed versus predicted outcome for decompressive craniectomy: a population-based study. J Neurotrauma. 2010; 27(7): 1225-1232, doi: 10.1089/neu.2010.1316, indexed in Pubmed: 20412014.

15. Marshall LF, Marshall SB, Klauber MR, et al. The diagnosis of head injury requires a classification based on computed axial tomography. J Neurotrauma. 1992; 9 Suppl 1: S287-S292, indexed in Pubmed: 1588618.

16. Maas AIR, Hukkelhoven CW, Marshall LF, et al. Prediction of outcome in traumatic brain injury with computed tomographic characteristics: a comparison between the computed tomographic classification and combinations of computed tomographic predictors. Neurosurgery. 2005; 57(6): 1173-82; discussion 1173, indexed in Pubmed: 16331165.

17. Huang YH, Deng YH, Lee TC, et al. Rotterdam computed tomography score as a prognosticator in head-injured patients undergoing decompressive craniectomy. Neurosurgery. 2012; 71(1): 80-85, doi: 10.1227/NEU.0b013e3182517aa1, indexed in Pubmed: 22382208.

18. Honeybul S, Ho KM, Lind CRP, et al. Validation of the CRASH model in the prediction of 18-month mortality and unfavorable outcome in severe traumatic brain injury requiring decompressive craniectomy. J Neurosurg. 2014; 120(5): 1131-1137, doi: 10.3171/2014.1.JNS131559, indexed in Pubmed: 24605836.

19. Wong GK, Teoh J, Yeung J, et al. Outcomes of traumatic brain injury in Hong Kong: validation with the TRISS, CRASH, and IMPACT models. J Clin Neurosci. 2013; 20(12): 1693-1696, doi: 10.1016/j. jocn.2012.12.032, indexed in Pubmed: 23993210.

20. Egea-Guerrero JJ, Rodríguez-Rodríguez A, Gordillo-Escobar E, et al. IMPACT Score for Traumatic Brain Injury: Validation of the Prognostic Tool in a Spanish Cohort. J Head Trauma Rehabil. 2018; 33(1): 46-52, doi: 10.1097/HTR.0000000000000292, indexed in Pubmed: 28195955. 
21. Panczykowski DM, Puccio AM, Scruggs BJ, et al. Prospective independent validation of IMPACT modeling as a prognostic tool in severe traumatic brain injury. J Neurotrauma. 2012; 29(1): 47-52, doi: 10.1089/ neu.2010.1482, indexed in Pubmed: 21933014.

22. Hukkelhoven CW, Steyerberg EW, Habbema JD, et al. Predicting outcome after traumatic brain injury: development and validation of a prognostic score based on admission characteristics. J Neurotrauma. 2005; 22(10): 1025-1039, doi: 10.1089/neu.2005.22.1025, indexed in Pubmed: 16238481.

23. Honeybul S, Ho KM. Predicting long-term neurological outcomes after severe traumatic brain injury requiring decompressive craniectomy: A comparison of the CRASH and IMPACT prognostic models. Injury. 2016; 47(9): 1886-1892, doi: 10.1016/j.injury.2016.04.017, indexed in Pubmed: 27157985.

24. Fraga $H$, Dourado I, Fernandes $R$, et al. Factores asociados a la incapacidad funcional global luego de transcurrido un año después del traumatismo craneoencefálico. Salud Colectiva. 2013; 9(3): 335, doi: 10.18294/sc.2013.190.

25. Andelic N, Sigurdardottir S, Arango-Lasprilla JC, et al. Long-Term Functional and Psychosocial Consequences and Health Care Provision after Traumatic Brain Injury. Behav Neurol. 2016; 2016: 2678081, doi: 10.1155/2016/2678081, indexed in Pubmed: 27034584.

26. Junqué C. Secuelas neuropsicológicas de los traumatismos craneoencefálicos. Revista de neurología. 1999; 28: 423-9.

27. The effectiveness of psychological interventions for aggressive behavior following acquired brain injury: A meta-analysis and systematic review.

28. Effective return-to-work interventions after acquired brain injury: A systematic review.

29. A manual-based vocational rehabilitation program for patients with an acquired brain injury: study protocol of a pragmatic randomized controlled trial (RCT). 